\title{
Comparison of intraocular pressure fluctuations before and after ab interno trabeculectomy in pseudoexfoliation glaucoma patients
}

This article was published in the following Dove Press journal:

Clinical Ophthalmology

14 September 2017

Number of times this article has been viewed

\author{
Naoki Tojo \\ Shinya Abe \\ Mari Miyakoshi \\ Atsushi Hayashi \\ Department of Ophthalmology, \\ Graduate School of Medicine and \\ Pharmaceutical Sciences, University \\ of Toyama, Toyama, Japan
}

Correspondence: Atsushi Hayashi Department of Ophthalmology, Graduate School of Medicine and Pharmaceutical Science, University of Toyama, 2630 Sugitani, Toyama, 930-0194, Japan

$\mathrm{Tel}+8 \mathrm{I} 764347363$

Fax +8I 764345037

Email ganka@med.u-toyama.ac.jp
Purpose: Ab interno trabeculectomy (AIT) with the Trabectome has been shown to reduce intraocular pressure (IOP) in eyes with pseudoexfoliation (PEX) glaucoma. Here, we examined the change of IOP fluctuations before and after only AIT or AIT with cataract surgery in PEX patients using the contact lens sensor Triggerfish ${ }^{\circledR}$.

Methods: This was a prospective open-label study. Twenty-four consecutive patients with PEX glaucoma were included. Twelve patients underwent cataract surgery and AIT (triple-surgery group), and 12 patients underwent only AIT (single-surgery group). In each eye, IOP fluctuations over $24 \mathrm{~h}$ were measured with the contact lens sensor before and at 3 months after the surgery. We compared the change of IOP fluctuation before and after operation. We also evaluated the difference in IOP changes between the triple- and single-surgery groups.

Results: At 3 months after the surgeries, the mean IOP was significantly reduced from $23.5 \pm 6.5 \mathrm{mmHg}$ to $14.6 \pm 2.8 \mathrm{mmHg}$ in the single-surgery group and from $22.5 \pm 3.0 \mathrm{mmHg}$ to $11.5 \pm 2.9 \mathrm{mmHg}$ in the triple-surgery group. The mean IOP reduction rate was significantly higher in the triple-surgery group compared to the single-surgery group $(p=0.0358)$. In both groups, the mean range of IOP fluctuations was significantly decreased during nocturnal periods. The mean range of $24 \mathrm{~h}$ IOP fluctuations was decreased in the triple-surgery group $(p=0.00425)$, not in the single-surgery group ( $p=0.970)$.

Conclusion: Triple surgery could decrease IOP value and the IOP fluctuations to a greater extent than single surgery in PEX glaucoma patients.

Keywords: pseudoexfoliation, ab interno trabeculectomy, contact lens sensor, fluctuation, intraocular pressure

\section{Introduction}

Glaucoma is a neurodegenerative disease of the eyes that leads to visual loss and ultimately blindness if left untreated. Pseudoexfoliation (PEX) glaucoma is one of the major causes of glaucoma in the elderly. ${ }^{1}$ It is estimated that about $25 \%$ of open-angle glaucoma are PEX glaucoma. ${ }^{2}$ It was reported that eyes with PEX show more rapid progression of glaucoma than those without PEX. ${ }^{2-4}$ PEX was reported to be a strong independent risk factor for glaucoma. ${ }^{2,5-8}$

Exfoliation materials accumulate on the front of capsule, the iris of pupillary margin, and Zinn's zonule. ${ }^{9}{ }^{10}$ The cause of intraocular pressure (IOP) increase in PEX glaucoma patients is thought to be due to blockage of the trabecular meshwork (TM) and trabecular cell dysfunction caused by exfoliation material accumulation. ${ }^{11}$

Some previous studies reported that PEX glaucoma patients had high IOP values and large IOP fluctuation. ${ }^{12,13}$ Other studies reported that large IOP fluctuations 
contribute to fast glaucoma progression. ${ }^{14-16}$ It might be essential to understand the time of peak IOP and IOP fluctuations for glaucoma management and treatment decisions. In order to understand peak IOP and IOP fluctuations more, many more number of measurements of IOP with Goldmann applanation tonometry (GAT) are required. Frequent measurement of IOP with GAT must overcome the burdens of hospitalization and sleep disturbance. The Triggerfish ${ }^{\circledR}$ (Sensimed, Lausanne, Switzerland) wireless contact lens sensor (CLS) has made it possible to measure habitual IOP fluctuations over $24 \mathrm{~h} \cdot{ }^{17,18}$

The CLS may provide better information about peak IOP and IOP fluctuations than measurements of temporary IOP during clinical hours alone.

$\mathrm{Ab}$ interno trabeculectomy (AIT) with a Trabcutome (NeoMedix, Tustin, CA, USA) has been reported as a potentially effective treatment for PEX. ${ }^{19}$ The performance of AIT with the Trabectome (which is an instrument used to permanently ablate a piece of TM and the Schlemm canal inner wall) can expose the collector channels directly to the aqueous in the anterior chamber. AIT can lower the IOP due to elimination of the outflow resistance of the TM and Schlemm canal inner wall. It was shown that AIT lowered the IOP and the number of antiglaucoma medications in PEX glaucoma patients. ${ }^{20}$ Francis et al ${ }^{21}$ also reported that combined phacoemulsification and intraocular lens implantation $(\mathrm{PEA}+\mathrm{IOL})$ and AIT lowered the IOP and medication use compared to the use of only AIT.

In this study, we measured IOP fluctuations using a CLS in patients with PEX glaucoma before and after AIT or AIT with cataract surgery, and we evaluated the effects of AIT on IOP fluctuations. We also compared the IOP values and the IOP fluctuations between AIT and AIT with cataract surgery groups.

\section{Methods}

\section{Subjects}

This was a prospective, open-label study. Twenty-four consecutive patients who were diagnosed with PEX glaucoma at Toyama University Hospital were enrolled. All subjects were recruited during the period from March 2015 to February 2016. All patients underwent a comprehensive ophthalmic examination including refraction, Goldmann gonioscopy, GAT, fundus examination, anterior-segment optical coherence tomography (CASIA SS-1000; Tomey, Nagoya, Japan), and automated perimetry (Humphrey Field Analyzer; Carl Zeiss Meditec, Dublin, CA, USA). A single glaucoma specialist diagnosed all the cases of PEX glaucoma.
The patients had already used tolerated glaucoma medications and had needed even more treatment to lower their IOP because of the progression of their visual field disorder. The research protocol was approved by the Institutional Review Board of the University of Toyama, and the procedures used conformed to the tenets of the Declaration of Helsinki. After the nature and possible consequences of the study were explained to the patients, written informed consent was obtained from each patient.

\section{Inclusion and exclusion criteria}

The diagnosis of PEX glaucoma was made if all four of the following criteria were satisfied: 1) the presence of white, flaky, dandruff-like material at the pupillary margin and/or on the anterior lens capsule; ${ }^{22}$ 2) the presence of glaucomatous optic disc neuropathy (a cup/disc ratio of $\geq 0.7$, or the presence of notching) accompanied by corresponding visual field defects; 3) a threshold examination of SITA 30-2 showing a glaucoma hemifield test result "outside normal limits," and a cluster of three contiguous points on the pattern deviation plot depressed at the $p<0.05$ level (occurring in age-matched normal subjects) not crossing the horizontal meridian that were compatible with glaucoma; and 4) a history of an IOP value of $>21 \mathrm{mmHg}$.

The inclusion criteria for the subjects were 1) best-corrected visual acuity $\geq 0.2,2$ ) spherical equivalent $<-6$ diopters and $<+6$ diopters, and 3) mean deviation $<-20$ decibel. We included patients who had a previous cataract surgery or selective laser trabeculoplasty at least 1 year earlier. Myopic eyes over -6 diopters were excluded before cataract surgery.

Patients were excluded if they had any of the following: 1) primary angle closure glaucoma, 2) neovascular glaucoma, 3) steroid glaucoma, 4) history of ocular trauma, 5) ocular inflammatory disease, and 6) prior glaucoma surgery.

\section{Measurement of IOP with GAT}

The baseline IOP was obtained by averaging the last three readings at almost the same time of the day prior to the AIT. We measured the postoperative IOP at 3 months after the surgeries in each patient at the same time of the day as that used at the baseline.

\section{Measurement of IOP with CLS}

Before and after the surgeries, each patient's $24 \mathrm{~h}$ continuous IOP was monitored. The CLS consisted of a highly oxygenpermeable soft contact lens with 2 sensing-resistive strain gauges that were capable of recording circumferential changes in the area of the corneoscleral junction. The circumferential 
changes in the area of the corneoscleral junction are assumed to correspond to IOP changes. ${ }^{18}$ The CLS can record for 30 seconds every 5 min (Figure 1A). The unit of measurement used in monitoring the IOP with the Triggerfish ${ }^{\circledR}$ is not millimeters of mercury but millivolt equivalent (mVeq), which is unique to Triggerfish ${ }^{\circledR}$. We started measuring the IOP fluctuation with CLS at about 9:00 a.m. for all patients. We also measured the IOP with GAT just before inserting the CLS and just after removing the CLS.

\section{Surgical techniques}

All patients were operated on by a single surgeon (NT) using the same surgical protocol as a previous report. ${ }^{19}$ All surgeries were performed under local (peribulbar) anesthesia.
A $1.6 \mathrm{~mm}$ temporal corneal incision at limbus was made, and the handpiece was injected into the anterior chamber. Under the Swan Jacob gonioscopic lens view, the tip of handpiece was inserted through the TM. Approximately $120^{\circ}$ arc of the TM and the inner wall of the Schlemm canal was ablated and removed. Patients with phakic eyes underwent cataract surgeries simultaneously. There was no case of lens preservation. A temporal clear cornel incision was made $(2.8 \mathrm{~mm})$, and conventional phacoemulsification and IOL implantation $($ PEA + IOL) were then performed. Phacoemulsification was performed with WHITESTAR SIGNATURE (Abbott Medical Optics, Santa Ana, CA, USA).

We separated the patients into two groups. The 12 patients who underwent only AIT comprised the single-surgery group.

A

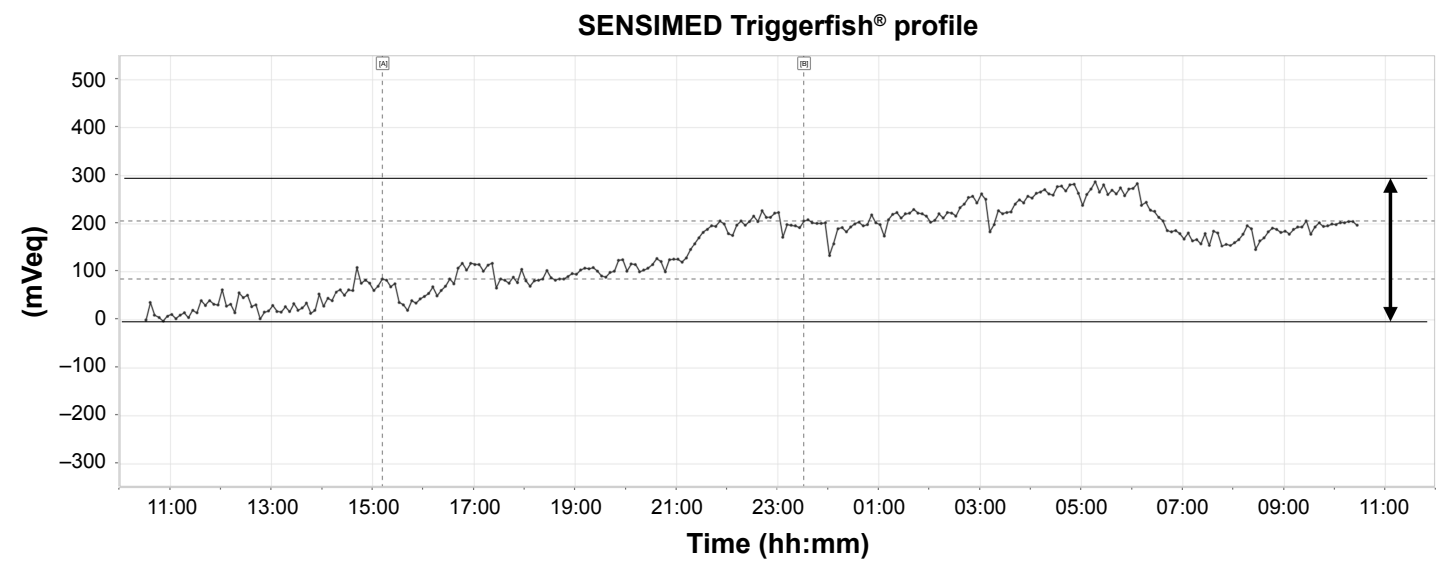

B

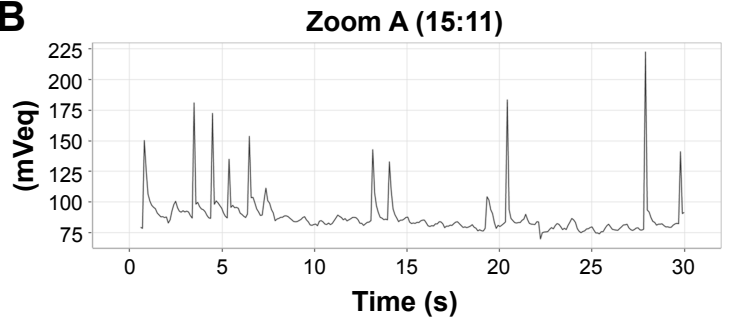

C

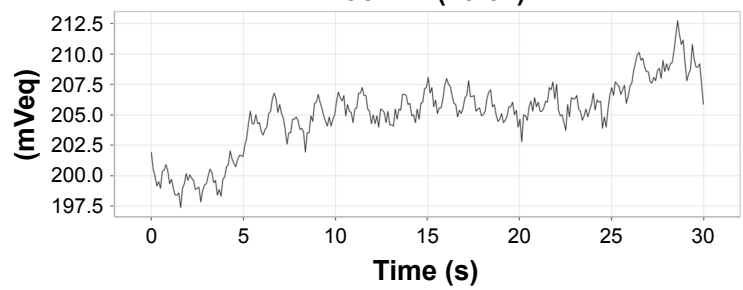

D

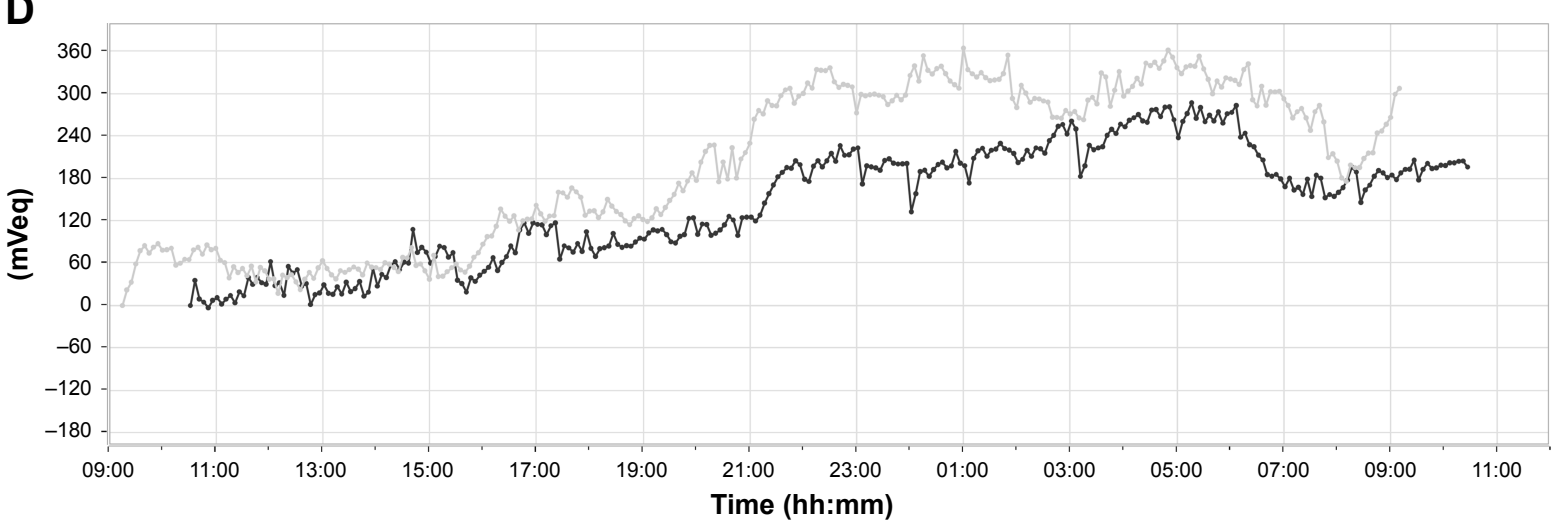

Figure I Example of the results of IOP fluctuation measurement over $24 \mathrm{~h}$ periods before and after AIT + PEA + IOL.

Notes: (A) Diagram of $24 \mathrm{~h}$ continuous IOP fluctuations using the Triggerfish ${ }^{\circledast} \mathrm{CLS}$. The difference between the maximum and minimum values (the arrow) was defined as the range of IOP fluctuations. (B) Zoom mode of IOP fluctuations during diurnal period. (C) Zoom mode of IOP fluctuations during nocturnal period. (D) Gray line: the IOP fluctuation before surgery. Black line: the IOP fluctuation after surgery.

Abbreviations: AIT, ab interno trabeculectomy; CLS, contact lens sensor; IOL, intraocular lens; IOP, intraocular pressure; PEA, phacoemulsification. 
The 12 patients who underwent AIT and PEA + IOL simultaneously comprised the triple-surgery group. We compared the IOP and the range of IOP fluctuations between the singleand triple-surgery groups.

\section{Postoperative medication}

Postoperative treatment was a topical combination of steroids and antibiotics that were reduced over 4 weeks following the intervention. In addition, $2 \%$ pilocarpine eye drops 4 times/ day were used for 4 weeks after surgery to avoid peripheral anterior synechia. The same antiglaucoma medication was continued throughout the study period.

\section{Evaluation of IOP fluctuations}

We evaluated IOP fluctuations separately in the diurnal and nocturnal periods as previously described. ${ }^{13}$ Since CLS could monitor accurately, we could see the spiking increase in IOP caused by eye blinks (Figure 1B) and the ocular pulsation frequency according to cardiac activity during sleep (Figure 1C). The nocturnal/sleep period was defined through the observation of blink eliminations as short and high spikes that were displayed by the software. We could easily recognize and separate sleeping and wakefulness with the software.

To analyze the IOP fluctuations, the range was defined as the difference between the maximum and minimum values over $24 \mathrm{~h}$, the diurnal period, and the nocturnal period (Figure 1A, D). To evaluate the patterns of IOP fluctuations for $24 \mathrm{~h}$ in each patient, the CLS signals were reconstituted by using the hourly mean IOP (mVeq).

\section{Evaluation of the change in corneal thickness and meridians}

The central corneal thickness (CCT) and corneal meridians, which may influence IOP measurement, were measured with anterior-segment optical coherence tomography before and after $24 \mathrm{~h}$ IOP measurement in each eye. The CCT, steeper meridian, and flatter meridian of all patients were measured twice before and after the surgeries with CASIA (Tomey Corp. Nagoya, Japan). We compared the differences before and after surgeries, and before and after the measurement of CLS. If there is a significant difference in the corneal curvature before and after the surgery, it will affect the data with CLS, so it is necessary to confirm.

\section{Definition of success}

Success was defined as when both of the following criteria were met: 1) postoperative IOP level between 6 and $21 \mathrm{mmHg}$, with or without antiglaucoma medications and 2) an IOP reduction $\geq 20 \%$ relative to the eye's preoperative value.
Eyes requiring additional glaucoma surgery that developed phthisis or that showed loss of light perception were classified as failures.

\section{Statistics}

A paired $t$-test and a Wilcoxon signed-rank test were used for all statistical analyses. Statistical significance was defined at $p<0.05$. Assuming that the standard deviation (100 $\mathrm{mVeq}$ ) of the IOP fluctuation was $100 \mathrm{mVeq}$, we found that a total of 10 pairs were necessary to detect a meaningful difference of $100 \mathrm{mVeq}$ with respect to the IOP fluctuation with $80 \%$ power and the two-sided significance level of 0.05 .

\section{Results \\ Ophthalmic data}

Table 1 shows the ophthalmic data of the patients. The study was performed on 24 eyes of 24 patients (15 males and 9 females: mean age $74.4 \pm 5.9$ years). No patients took oral acetazolamide. Twelve patients who had undergone PEA + IOL surgery underwent only AIT surgeries (singlesurgery group). The other 12 patients with phakic eye were operated with PEA + IOL + AIT triple surgery (triple-surgery group).

In one patient, Zinn's zonule was so loose that we could not place the IOL in the capsule; we thus performed a vitrectomy and IOL was fixed at the ciliary sulcus. Other surgeries were performed successfully, and no intraoperative complications occurred. After the surgeries, there were no severe postoperative complications such as hypotony, hypotony maculopathy, choroidal effusion or hemorrhage, and endophthalmitis.

Table I Ophthalmic data of the PEX patients

\begin{tabular}{lllll}
\hline Characteristic & $\begin{array}{l}\text { Total } \\
(\mathbf{n}=\mathbf{2 4})\end{array}$ & $\begin{array}{l}\text { Single } \\
(\mathbf{n}=\mathbf{I 2})\end{array}$ & $\begin{array}{l}\text { Triple } \\
(\mathbf{n}=\mathbf{I 2})\end{array}$ & p-value \\
\hline Age (years) & $74.4 \pm 5.9$ & $76.3 \pm 6.0$ & $72.4 \pm 5.4$ & 0.108 \\
Gender (male) & $15(62.5 \%)$ & $6(50 \%)$ & $9(75 \%)$ & 0.746 \\
CCT $(\mu \mathrm{m})$ & $526 \pm 36$ & $538 \pm 36$ & $515 \pm 35$ & 0.131 \\
Medication & $3.6 \pm 0.7$ & $3.6 \pm 0.7$ & $3.7 \pm 0.7$ & 0.689 \\
Pre-IOP $(\mathrm{mmHg})$ & $23.0 \pm 5.0$ & $23.5 \pm 6.5$ & $22.5 \pm 3.0$ & 0.653 \\
Post-IOP $(\mathrm{mmHg})$ & $13.0 \pm 3.3$ & $14.6 \pm 2.8$ & $1 \mathrm{I} .5 \pm 2.9$ & $\mathbf{0 . 0 1 5 4}$ \\
IOP reduction $(\%)$ & $41.5 \pm 15.9$ & $34.8 \pm 16.2$ & $48.2 \pm 13.0$ & $\mathbf{0 . 0 3 5 8}$ \\
\hline
\end{tabular}

Notes: Data are mean \pm standard error. Single: single-surgery group (only AIT). Triple: triple-surgery group (cataract surgery and AIT simultaneously). Pre-IOP: intraocular pressure before the surgeries. Post-IOP: intraocular pressure after the surgeries. IOP reduction: IOP reduction rate before and after surgeries. The $p$-value is the comparison between the single- and triple-surgery groups. Bold $p$-values are statistically significant $(p<0.05)$.

Abbreviations: AIT, ab interno trabeculectomy; CCT, central corneal thickness; IOP, intraocular pressure; PEX, pseudoexfoliation. 
Table 2 Comparison of IOP fluctuations before and after AIT surgery

\begin{tabular}{|c|c|c|c|c|c|c|c|c|c|}
\hline & \multicolumn{3}{|l|}{$24 \mathrm{~h}$} & \multicolumn{3}{|c|}{ Diurnal period } & \multicolumn{3}{|c|}{ Nocturnal period } \\
\hline & Preop & Postop & $p$-value & Preop & Postop & $p$-value & Preop & Postop & $p$-value \\
\hline All $(n=24)$ & $487 \pm 170$ & $428 \pm 139$ & 0.166 & $449 \pm 167$ & $429 \pm|3|$ & 0.761 & $277 \pm 129$ & $|90 \pm 6|$ & $<0.001$ \\
\hline Single $(n=12)$ & $498 \pm 208$ & $448 \pm 172$ & 0.97 & $465 \pm 205$ & $466 \pm 157$ & 0.519 & $305 \pm 162$ & $196 \pm 68$ & 0.0425 \\
\hline Triple $(n=12)$ & $476 \pm 130$ & $408 \pm 99$ & 0.0425 & $433 \pm 126$ & $392 \pm 92$ & 0.23 & $250 \pm 81$ & $185 \pm 55$ & 0.0049 \\
\hline
\end{tabular}

Notes: Data are mean \pm standard error. Preop: preoperative IOP fluctuation measured with CLS ( $\mathrm{mVeq}$ ). Postop: postoperative IOP fluctuation measured with CLS ( $\mathrm{mVeq}$ ). Single: single-surgery group (only AIT). Triple: triple-surgery group (cataract surgery and AIT simultaneously). Bold $p$-values are statistically significant.

Abbreviations: AIT, ab interno trabeculectomy; CLS, contact lens sensor; IOP, intraocular pressure.

\section{IOP values before and after the surgeries}

The mean preoperation IOP values of the two groups were not significantly different $(p=0.653)$. The mean IOP values at 3 months were significantly decreased from $23.5 \pm 6.5 \mathrm{mmHg}$ to $14.6 \pm 2.8 \mathrm{mmHg}$ in the single-surgery group ( $p=0.0002)$ and from $22.5 \pm 3.0 \mathrm{mmHg}$ to $11.5 \pm 2.9$ $\mathrm{mmHg}$ in the triple-surgery group $(p=0.0005)$. The mean IOP reduction rate was $41.5 \% \pm 15.9 \%$ in all patients. The IOP reduction rate was significantly higher in the triple-surgery group (48.2\% $\pm 13.0 \%$ ) compared to the single-surgery group $(34.8 \% \pm 16.2 \%)(p=0.0358)$. The success rate in the singlesurgery group was $83.3 \%$, and there were two cases in which the IOP reduction rate was lower than $20 \%$. The success rate in the triple-surgery group was $100 \%$. There was no case of additional operation within 3 months.

\section{Measurement of IOP fluctuations with CLS}

Habitual IOP fluctuations over $24 \mathrm{~h}$ in each patient were successfully recorded using a CLS. The patients showed no serious complications related to measurement with the CLS. Table 2 shows the results of IOP fluctuations with CLS before and after surgeries. The mean range of IOP fluctuations over $24 \mathrm{~h}$ and during the diurnal periods was not significantly different $(p=0.166, p=0.761)$. In contrast, during the nocturnal periods, statistical significance was observed $(p<0.001)$. The ranges of IOP fluctuations before and after single surgery are shown in Figure 2. Similarly, IOP fluctuations decreased significantly during the nocturnal periods $(p=0.0425)$. IOP fluctuations during the nocturnal period decreased in 10 of the 12 AIT-alone patients. The ranges of IOP fluctuations in the triple-surgery group are illustrated in Figure 3. The mean range of IOP fluctuations over $24 \mathrm{~h}$ and during the nocturnal periods was significantly decreased $(p=0.0425, p=0.0049)$. The IOP fluctuations over $24 \mathrm{~h}$ and during the nocturnal periods decreased in 9 of the 12 patients.

Figure 4 shows the average of IOP fluctuations at each hour-long period before and after AIT. The IOP tended to rise gradually from noon to nighttime and fall during early morning. On average, the IOP (mVeq) fell rapidly at 5:00 a.m. before AIT. The IOP fell more gently after the AIT. Figure 5 shows the average of IOP fluctuations at each hour-long period before and after AIT + PEA + IOL. The range of IOP fluctuations was smaller throughout the day, especially during nocturnal periods, after the surgeries. Figure 1D shows a typical example of IOP fluctuations before and after triple surgery.

\section{Corneal effects of the CLS}

To examine the effects of the CLS on the cornea, we analyzed CCT, steeper meridian, and flatter meridian. We compared these items before and after surgeries, and all items showed no significant difference (Table 3).

We also compared these items between before and after measurement with the CLS (Table 4). All items were largely unchanged.

\section{Discussion}

We performed continuous $24 \mathrm{~h}$ IOP fluctuation monitoring before and after surgeries in 24 eyes with PEX glaucoma. Both single and triple surgeries resulted in significant

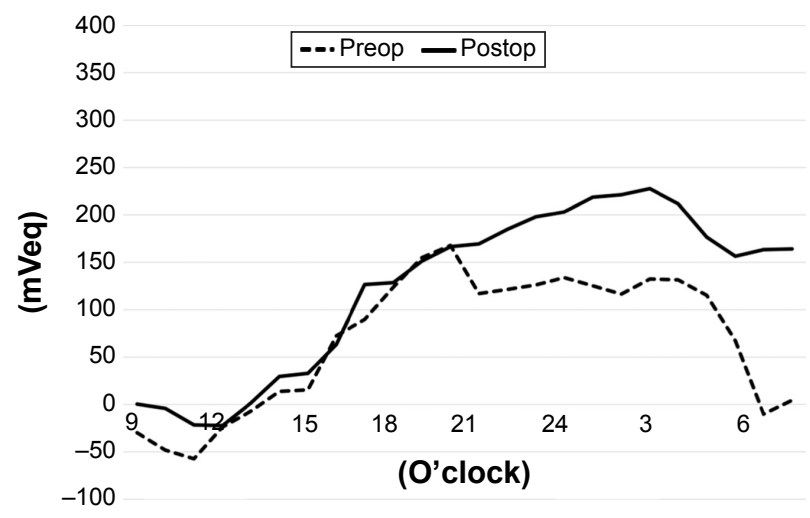

Figure 2 The average of IOP fluctuation at each I-hr period before and after AIT (single-surgery group).

Notes: Dotted line: before the AIT surgery. Solid line: After AIT surgery. Before AIT, the line of falls rapidly at 5:00 a.m. The IOP fluctuation was smaller before AIT than after AIT during the nocturnal periods.

Abbreviations: AIT, ab interno trabeculectomy; IOP, intraocular pressure. 


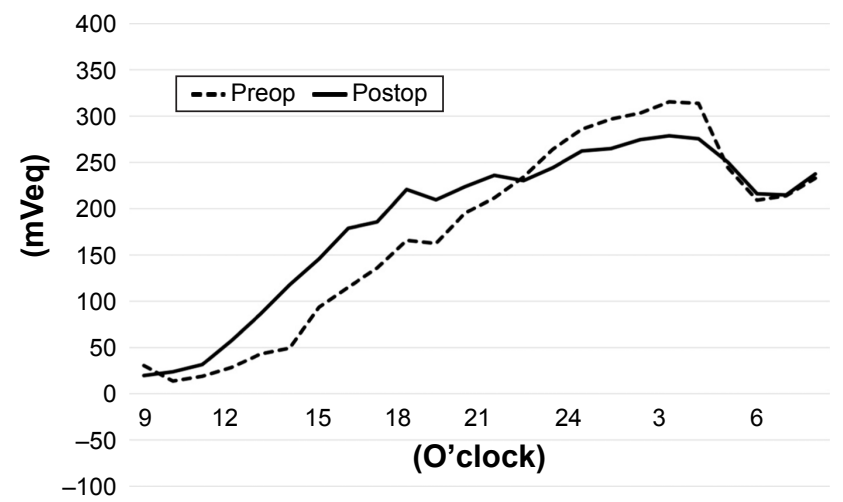

Figure 3 The average of IOP fluctuation at each I hr period before and after AIT + PEA + IOL (triple-surgery group).

Notes: Dotted line: before the AIT + PEA + IOL. Solid line: After the surgeries. Abbreviations: AIT, ab interno trabeculectomy; IOL, intraocular lens; IOP, intraocular pressure; PEA, phacoemulsification.

decreases in the IOP values and IOP fluctuations during the nocturnal periods after 3 months.

Klamann reported that AIT was effective in IOP reduction in cases of PEX glaucoma. ${ }^{18}$ Other studies showed that triple surgery more effectively lowered IOP compared to only single surgery. ${ }^{19,23}$ Our present findings agreed with these studies' results. In our investigation, single surgery for PEX glaucoma patients resulted in improved IOP, and triple surgery was more effective at lowering IOP than single surgery. The IOP reduction rate in previous reports was approximately $37.3 \%-39.3 \%$ at 3 months after AIT surgeries. ${ }^{17,18}$ These data are almost equal to our result. Parikh et $\mathrm{al}^{25}$ reported

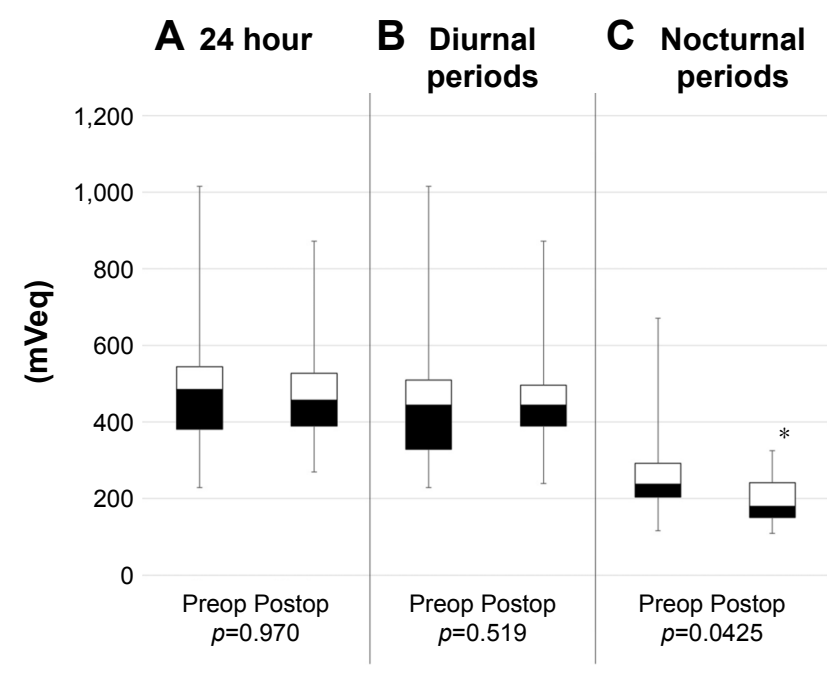

Figure 4 The range of IOP fluctuation in single-surgery group.

Notes: Boxplots of the range of IOP fluctuation (A) over $24 \mathrm{hr}$ and during the (B) diurnal periods and $(\mathbf{C})$ nocturnal periods before and after AIT. AIT significantly decreased the range of IOP fluctuation during the nocturnal periods $(p=0.0425)$. $*_{p}<0.05$.

Abbreviations: AIT, ab interno trabeculectomy; IOP, intraocular pressure.

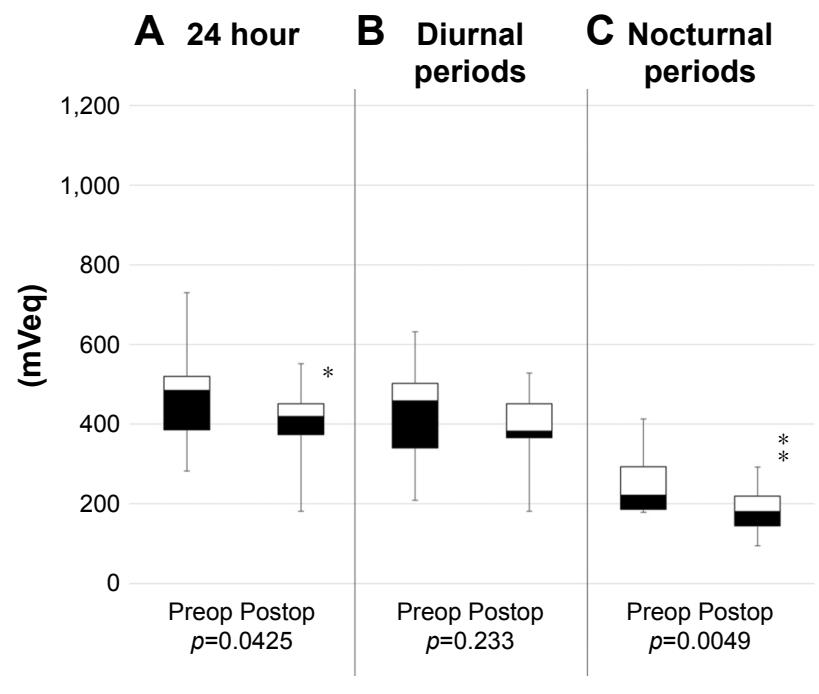

Figure 5 The range of IOP fluctuation in Triple-surgery group.

Notes: Boxplots of the range of IOP fluctuation (A) over 24-hr and during the (B) diurnal periods and (C) nocturnal periods before and after AIT + PEA + IOL. $\mathrm{AIT}+\mathrm{PEA}+\mathrm{IOL}$ significantly decreased the range of IOP fluctuation throughout the day $(p=0.0425)$ and during the nocturnal periods $(p=0.0049) . * 0.05 ; * * 0.01$.

Abbreviations: AIT, ab interno trabeculectomy; IOL, intraocular lens; IOP, intraocular pressure; PEA, phacoemulsification.

that PEA + IOL did not make a significant contribution to postoperative IOP when combined with trabectome surgery in phakic patients.

We suspected that there are three reasons for the greater reduction in mean IOP after triple surgery compared to single surgery. The first reason is the opening of the anterior chamber angle, and the second reason is the effect of washout of exfoliation material. The third reason is that phacoemulsification could make the front surface of the lens move backward, and the ciliary muscle to relax, and so the TM and Schlemm's canal stretch and the aqueous flow is improved. Bussel et $\mathrm{al}^{25}$ reported that the width of the preoperative angle was not associated with the results of AIT + PEA + IOL surgeries.

In PEX glaucoma, the aqueous flow resistance of TM is related to the accumulation of exfoliation materials. When patients undergo PEA + IOL, irrigation and aspiration could wash out the exfoliation materials. Damji et $\mathrm{al}^{26}$ reported that PEA + IOL could decrease the IOP more in patients with PEX compared to those without PEX. Widder et $\mathrm{al}^{23}$ observed that trabecular aspiration could decrease IOP in PEX glaucoma patients.

Whether laser trabeculoplasty is a factor in AIT failure has not been established..$^{27,28}$ Due to the relatively few cases in our present study, we could not address this issue.

Because the reproducibility of the measurement of the circadian IOP pattern with the CLS has been confirmed to be acceptable, ${ }^{29,30}$ we used the CLS to examine changes in 
Table 3 Change in curvatures before and after AIT surgery

\begin{tabular}{|c|c|c|c|c|c|c|}
\hline & \multicolumn{3}{|l|}{ Single } & \multicolumn{3}{|l|}{ Triple } \\
\hline & Preop & Postop & $p$-value & Preop & Postop & $p$-value \\
\hline $\mathrm{CCT}(\mu \mathrm{m})$ & $538 \pm 36$ & $532 \pm 32$ & 0.163 & $512 \pm 32$ & $517 \pm 28$ & 0.113 \\
\hline Steeper meridian $(D)$ & $44 . I \pm I .7$ & $44.1 \pm 1.5$ & 0.992 & $44.5 \pm 1.5$ & $44.7 \pm 1.6$ & 0.482 \\
\hline Flatter meridian (D) & $44.7 \pm 1.7$ & $43.7 \pm 1.6$ & 0.117 & $45.0 \pm 1.9$ & $44.8 \pm 2.1$ & 0.656 \\
\hline
\end{tabular}

Notes: Data are mean \pm standard error. Single: single-surgery group (only AIT). Triple: triple-surgery group (cataract surgery and AIT simultaneously).

Abbreviations: AIT, ab interno trabeculectomy; CCT, central corneal thickness.

IOP fluctuations before and after single or triple surgery. Considering that the glaucoma medication was not changed, and the corneal curvature was not significantly different between before and after surgeries, we thought that the IOP fluctuations with CLS were measured on almost the same conditions. Our analysis of IOP fluctuations with the CLS in both patient groups demonstrated that IOP fluctuations were reduced during the nocturnal period. We observed that $79.2 \%$ (19 of 24) of the PEX glaucoma patients had decreased IOP fluctuations during the nocturnal periods. This improvement of IOP fluctuations might be important for the prevention of glaucoma progression in PEX glaucoma patients, because IOP fluctuations have been associated with a progressive deterioration of the visual field. ${ }^{31,32} \mathrm{We}$ had compared the fluctuation of IOP with CLS between normal-tension glaucoma patients and healthy subjects. Although there was no significant difference in the mean of IOP, the IOP fluctuation was significantly larger in the normal-tension glaucoma patients. ${ }^{33}$ The volume of IOP fluctuation and the value of IOP might be independent factors. Some of studies reported that the IOP fluctuation and the progression of glaucoma were related, but this remains controversial. ${ }^{34,35}$ The reason for the decrease in IOP fluctuation might be due to improvement of outflow from the TM. Since the outflow from the TM increases as the IOP increases, it might be considered that IOP fluctuations could be decreased. Triple surgery could further open the angle, so the flow from TM is likely to increase, so IOP fluctuation becomes smaller. The reason why the IOP fluctuation becomes small during nocturnal periods is not yet known.

In addition, peak IOP is known to be a risk factor for glaucoma progression. ${ }^{7}$ Although we could not determine the

Table 4 Change in curvatures before and after measurement with the CLS

\begin{tabular}{llll}
\hline & $\begin{array}{l}\text { Before } \\
\text { measurement }\end{array}$ & $\begin{array}{l}\text { After } \\
\text { measurement }\end{array}$ & p-value \\
\hline CCT $(\mu \mathrm{m})$ & $526 \pm 36$ & $528 \pm 41$ & 0.125 \\
Steeper meridian (D) & $44.2 \pm 1.7$ & $43.8 \pm 1.9$ & 0.136 \\
Flatter meridian (D) & $43.1 \pm 1.6$ & $42.8 \pm 1.9$ & 0.0593 \\
\hline
\end{tabular}

Note: Data are mean \pm standard error.

Abbreviations: CCT, central corneal thickness; CLS, contact lens sensor. exact peak IOP in units of $\mathrm{mmHg}$, CLS measurement showed the time of the peak IOP in the course of the $24 \mathrm{~h}$ habitual IOP fluctuations. Barkana et $\mathrm{al}^{36}$ and Jonas et $\mathrm{al}^{37}$ reported that $69 \%-75 \%$ of glaucoma patients had peak IOP outside regular clinic hours. In the present study, 83.3\% (20 of 24) of the PEX patients had the preoperative maximum IOP value outside clinic hours. All of the postoperative maximum IOP values were during nocturnal periods. Measurements of IOP during clinic hours may fail to reveal the individual's true IOP fluctuations. We had compared the fluctuations of IOP with CLS between PEX glaucoma patients and healthy subjects, and PEX glaucoma patients were found to have higher IOP fluctuation than healthy sujects. ${ }^{13} \mathrm{We}$ found that CLS has advantages for examining peak IOP and IOP fluctuation throughout the day.

The possibility that artifacts with CLS might affect our results cannot be neglected. Hubanova compared the eyes between with CLS and without CLS (the control eyes), Wearing of the CLS overnight made CCT and corneal curvature irregularities increase significantly. ${ }^{38}$ Freiberg et al ${ }^{39}$ reported that wearing CLS did not affect the CCT, although the CLS induced some corneal swelling. In our study, the CCT was not significantly different before and after the measurement with CLS. The anterior steeper and anterior flatter meridians ware smaller. The reason might be due to the effects caused by gluing the CLS to the corneal surface.

Nonetheless, the measurement of IOP with the CLS has some limitations. The first limitation is that it is not possible to convert the recorded units of $\mathrm{mVeq}$ to units of $\mathrm{mmHg}$. The CLS records relative IOP from the initial IOP, not the absolute IOP. The range of IOP fluctuations in PEX glaucoma is $\sim 4.8 \mathrm{mmHg}$ with GAT. ${ }^{40}$ The relationship between the CLS device output and IOP as measured with GAT is known to be proportional, but not convert the units. The evaluation of CLS needs further study at present. Leonardi et $\mathrm{al}^{18}$ demonstrated that the IOP and the output of CLS were directly proportional. ${ }^{21}$ Mansouri et $\mathrm{al}^{30}$ reported that the reproducibility with CLS was good. Considering the measurement, the same patient, and no change in glaucoma 
medication, the large IOP fluctuation measured with CLS might reflect real IOP changes.

A second limitation is the effects of the changes in the corneal shape. Wearing a CLS for $24 \mathrm{~h}$ might influence the corneal shape and the value of mVeq. In our study, CCT and corneal curvature were not significantly different before and after CLS measurement. A further study on CLS is needed to elucidate the relation of value of CLS and corneal shape.

A third limitation is that we did not consider long-term IOP fluctuations. A greater IOP fluctuation range and high maximum IOP levels may be contributory factors in PEX glaucoma. In this regard, treatment for glaucoma is needed not only to reduce temporary IOP but also to decrease the IOP fluctuation. Even though there are some limitations of the CLS, it is useful to continuously measure IOP fluctuation. Examination of IOP fluctuation with the CLS may become a useful tool for glaucoma treatment.

The measurement of IOP fluctuation with CLS is an accurate and reproducible method, and we found it useful to evaluate the IOP fluctuation. Both the AIT and AIT + PEA + IOL surgeries resulted in a decrease in IOP and IOP fluctuation during nocturnal periods. Triple surgery resulted in greater decreases in IOP value and IOP fluctuation compared to single surgery after 3 months. CLS might thus become a useful tool to evaluate IOP fluctuations in future.

\section{Disclosure}

The authors report no conflicts of interest in this work.

\section{References}

1. Ritch R, Schlotzer-Schrehardt U. Exfoliation syndrome. Surv Ophthalmol. 2001;45(4):265-315.

2. Mitchell P, Wang JJ, Hourihan F, Healey PR, Mitchell P. The relationship between glaucoma and pseudoexfoliation: the Blue Mountains Eye Study. Arch Ophthalmol. 1999;117(10):1319-1324.

3. Ritch R, Schlotzer-Schrehardt U. Exfoliation (pseudoexfoliation) syndrome: toward a new understanding. Proceedings of the First International Think Tank. Acta Ophthalmol Scand. 2001;79(2):213-217.

4. Teus MA, Castejon MA, Calvo MA, Pérez-Salaíces P, Marcos A. Intraocular pressure as a risk factor for visual field loss in pseudoexfoliative and in primary open-angle glaucoma. Ophthalmology. 1998;105(12): 2225-2229.

5. Grodum K, Heijl A, Bengtsson B. Risk of glaucoma in ocular hypertension with and without pseudoexfoliation. Ophthalmology. 2005;112(3): 386-390.

6. De Moraes CG, Juthani VJ, Liebmann JM, et al. Risk factors for visual field progression in treated glaucoma. Arch Ophthalmol. 2011; 129(5):562-568.

7. Gardiner SK, Johnson CA, Demirel S. Factors predicting the rate of functional progression in early and suspected glaucoma. Invest Ophthalmol Vis Sci. 2012;53(7):3598-3604.

8. Konstas AG, Quaranta L, Mikropoulos DG, et al. Peak intraocular pressure and glaucomatous progression in primary open-angle glaucoma. J Ocul Pharmacol Ther. 2012;28(1):26-32.
9. Schlotzer-Schrehardt U, Naumann GO. Ocular and systemic pseudoexfoliation syndrome. Am J Ophthalmol. 2006;141(5):921-937.

10. Kuchle M, Vinores SA, Mahlow J, Green WR. Blood-aqueous barrier in pseudoexfoliation syndrome: evaluation by immunohistochemical staining of endogenous albumin. Graefes Arch Clin Exp Ophthalmol. 1996;234(1):12-18.

11. Ahrlich KG, De Moraes CG, Teng CC, et al. Visual field progression differences between normal-tension and exfoliative high-tension glaucoma. Invest Ophthalmol Vis Sci. 2010;51(3):1458-1463.

12. De Moraes CG, Liebmann JM, Liebmann CA, Susanna R Jr, Tello C, Ritch R. Visual field progression outcomes in glaucoma subtypes. Acta Ophthalmol. 2013;91(3):288-293.

13. Tojo N, Hayashi A, Otsuka M, Miyakoshi A. Fluctuations of the intraocular pressure in pseudoexfoliation syndrome and normal eyes measured by a contact lens sensor. J Glaucoma. 2016;25(5):e463-e468.

14. Komori S, Ishida K, Yamamoto T. Results of long-term monitoring of normal-tension glaucoma patients receiving medical therapy: results of an 18-year follow-up. Graefes Arch Clin Exp Ophthalmol. 2014;252(12):1963-1970.

15. Musch DC, Gillespie BW, Niziol LM, et al; CIGTS Study Group. Intraocular pressure control and long-term visual field loss in the Collaborative Initial Glaucoma Treatment Study. Ophthalmology. 2011; 118(9):1766-1773.

16. Nouri-Mahdavi K, Hoffman D, Coleman AL, et al; Advanced Glaucoma Intervention Study. Predictive factors for glaucomatous visual field progression in the Advanced Glaucoma Intervention Study. Ophthalmology. 2004;111(9):1627-1635.

17. Mansouri K, Shaarawy T. Continuous intraocular pressure monitoring with a wireless ocular telemetry sensor: initial clinical experience in patients with open angle glaucoma. Br J Ophthalmol. 2011;95(5):627-629.

18. Leonardi M, Pitchon EM, Bertsch A, Renaud P, Mermoud A. Wireless contact lens sensor for intraocular pressure monitoring: assessment on enucleated pig eyes. Acta Ophthalmol. 2009;87(4):433-437.

19. Ting JL, Damji KF, Stiles MC; Trabectome Study Group. Ab interno trabeculectomy: outcomes in exfoliation versus primary open-angle glaucoma. J Cataract Refract Surg. 2012;38(2):315-323.

20. Klamann MK, Gonnermann J, Maier AK, et al. Combined clear cornea phacoemulsification in the treatment of pseudoexfoliative glaucoma associated with cataract: significance of trabecular aspiration and ab interno trabeculectomy. Graefes Arch Clin Exp Ophthalmol. 2013;251(9): 2195-2199.

21. Francis BA, Minckler D, Dustin L, et al. Combined cataract extraction and trabeculotomy by the internal approach for coexisting cataract and open-angle glaucoma: initial results. J Cataract Refract Surg. 2008; 34(7):1096-1103.

22. Thomas R, Nirmalan PK, Krishnaiah S. Pseudoexfoliation in southern India: the Andhra Pradesh Eye Disease Study. Invest Ophthalmol Vis Sci. 2005;46(4):1170-1176.

23. Widder RA, Dinslage S, Rosentreter A, et al. A new surgical triple procedure in pseudoexfoliation glaucoma using cataract surgery, Trabectome, and trabecular aspiration. Graefes Arch Clin Exp Ophthalmol. 2014;252(12):1971-1975.

24. Parikh HA, Bussel II, Schuman JS, Brown EN, Loewen NA. Coarsened exact matching of phaco-trabectome to trabectome in phakic patients: lack of additional pressure reduction from phacoemulsification. PLoS One. 2016;11(2):e0149384.

25. Bussel II, Kaplowitz K, Schuman JS, Loewen NA; Trabectome Study Group. Outcomes of ab interno trabeculectomy with the trabectome by degree of angle opening. Br J Ophthalmol. 2015;99(7):914-919.

26. Damji KF, Konstas AG, Liebmann JM, et al. Intraocular pressure following phacoemulsification in patients with and without exfoliation syndrome: a 2 year prospective study. Br J Ophthalmol. 2006;90(8): 1014-1018.

27. Ahuja Y, Ma Khin Pyi S, Malihi M, Hodge DO, Sit AJ. Clinical results of ab interno trabeculotomy using the trabectome for open-angle glaucoma: the Mayo Clinic series in Rochester, Minnesota. Am J Ophthalmol. 2013; 156(5):927-935. 
28. Klamann MK, Gonnermann J, Maier AK, Bertelmann E, Joussen AM, Torun N. Influence of Selective Laser Trabeculoplasty (SLT) on combined clear cornea phacoemulsification and Trabectome outcomes. Graefes Arch Clin Exp Ophthalmol. 2014;252(4):627-631.

29. Mansouri K, Liu JH, Weinreb RN, Tafreshi A, Medeiros FA. Analysis of continuous 24-hour intraocular pressure patterns in glaucoma. Invest Ophthalmol Vis Sci. 2012;53(13):8050-8056.

30. Mansouri K, Medeiros FA, Tafreshi A, Weinreb RN. Continuous 24-hour monitoring of intraocular pressure patterns with a contact lens sensor: safety, tolerability, and reproducibility in patients with glaucoma. Arch Ophthalmol. 2012;130(12):1534-1539.

31. Chiselita D, Motoc I, Danielescu C. Daily and nightly fluctuation of intraocular pressure and blood pressure in glaucoma and non-glaucoma patients. Oftalmologia. 2008;52(1):119-125.

32. Altintas O, Yuksel N, Karabas VL, Qağlar Y. Diurnal intraocular pressure variation in pseudoexfoliation syndrome. Eur J Ophthalmol. 2004; 14(6):495-500.

33. Tojo N, Abe S, Ishida M, Yagou T, Hayashi A. The fluctuation of intraocular pressure measured by a contact lens sensor in normal-tension glaucoma patients and nonglaucoma subjects. J Glaucoma. 2017;26(3): 195-200.

34. Bengtsson B, Leske MC, Hyman L, Heijl A; Early Manifest Glaucoma Trial Group. Fluctuation of intraocular pressure and glaucoma progression in the early manifest glaucoma trial. Ophthalmology. 2007;114(2):205-209.
35. Medeiros FA, Weinreb RN, Zangwill LM, et al. Long-term intraocular pressure fluctuations and risk of conversion from ocular hypertension to glaucoma. Ophthalmology. 2008;115(6):934-940.

36. Barkana Y, Anis S, Liebmann J, Tello C, Ritch R. Clinical utility of intraocular pressure monitoring outside of normal office hours in patients with glaucoma. Arch Ophthalmol. 2006;124(6):793-797.

37. Jonas JB, Budde W, Stroux A, Oberacher-Velten IM, Jünemann A Single intraocular pressure measurements and diurnal intraocular pressure profiles. Am J Ophthalmol. 2005;139(6):1136-1137.

38. Hubanova R, Aptel F, Zhou T, et al. Comparison of intraocular pressure measurements with the Reichert Pt100, the Keeler Pulsair Intellipuff portable noncontact tonometers, and Goldmann applanation tonometry. J Glaucoma. 2015;24(5):356-363.

39. Freiberg FJ, Lindell J, Thederan LA, Leippi S, Shen Y, Klink T. Corneal thickness after overnight wear of an intraocular pressure fluctuation contact lens sensor. Acta Ophthalmol. 2012;90(7):e534-e539.

40. Gumus K, Bozkurt B, Sonmez B, Irkec M, Orhan M, Saracbasi O. Diurnal variation of intraocular pressure and its correlation with retinal nerve fiber analysis in Turkish patients with exfoliation syndrome. Graefes Arch Clin Exp Ophthalmol. 2006;244(2):170-176.
Clinical Ophthalmology

\section{Publish your work in this journal}

Clinical Ophthalmology is an international, peer-reviewed journal covering all subspecialties within ophthalmology. Key topics include: Optometry; Visual science; Pharmacology and drug therapy in eye diseases; Basic Sciences; Primary and Secondary eye care; Patien Safety and Quality of Care Improvements. This journal is indexed on

Submit your manuscript here: http://www.dovepress.com/clinical-ophthalmology-journal

\section{Dovepress}

PubMed Central and CAS, and is the official journal of The Society of Clinical Ophthalmology (SCO). The manuscript management system is completely online and includes a very quick and fair peer-review system, which is all easy to use. Visit http://www.dovepress.com/ testimonials.php to read real quotes from published authors. 